\title{
Gun Dynamic Parameters Measurement Technology on Live Firing Condition
}

\author{
QIN Jun-qi ${ }^{1, \text { a }}$,YANG Yu-liang ${ }^{1}$, DI Chang-chun ${ }^{1}$, WANG Dong ${ }^{2}$ \\ (1 Ordnance Engineering College, Shijiazhuang 050003, China \\ 2 Army of aviation institute, Beijing, 101121, China) \\ ayyl_liang@sina.com
}

Keywords: live firing; measurement technology; recoil displacement; gun carriage stress

\begin{abstract}
Recoil displacement, recoil velocity, recoil resistance and gun carriage stress were selected as the key dynamic parameters. Measurement programs were developed respectively according to the characteristics of dynamic parameters. Live firing test was carried out, and measurement results of dynamic parameters were filtered by the low-pass filtering and EMD method. Finally, smooth test curves of dynamic parameters were obtained. This research can provide a method reference for the measurement technology and data processing of gun test.
\end{abstract}

\section{Introduction}

Gun live firing was a very complex dynamic process with intense vibration, which brought a great inconvenience for the gun test measuring. The improvement of gun informatization level also required that the measurement technology of dynamic parameters should be efficient, intelligent, portable and intensive. Therefore, the paper selected four common dynamic parameters, and carried out study on measurement technology and test data processing.

\section{Measure programs of dynamic parameters}

\section{Measurement program of recoil displacement}

Measurement sensors of recoil displacement mainly consisted of the steel-belt speedometer, the photoelectric steel-belt speedometer and the cable-type displacement sensor ${ }^{[1]}$. The cable-type displacement sensor was the most widely used for the advantages of small volume, low mass and high precision ${ }^{[2]}$.

On the measurement process, the body part of the displacement sensor was fixed on the inertial navigation system, and the cable part the displacement sensor was connected to the breech. The displacement sensor type was PT5DC, with the range of 50 inches.

\section{Measurement program of recoil resistance}

Recoil resistance mainly consisted of three parts, the recuperator force, the recoil brake force and the constant resistance ${ }^{[3]}$. According to the composition of recoil resistance, the measurement program was set up.

(1) The initial gas pressure of recuperator was measured by the pressure gauge of PY-100 type with the range of $10 \mathrm{MPa}$.

(2) Each chamber pressure of recoil brake was measured by the piezoelectric pressure sensor KISTLER 7005 with the range of 60MPa.

(3) The firing angle was measured by the quadrant.

\section{Measurement program of gun carriage stress}

Gun carriage stress was measured by the resistance strain gauge. Resistance strain gauge can convert the stress change into the resistance change. The stress test principle was shown in Figure 1. 


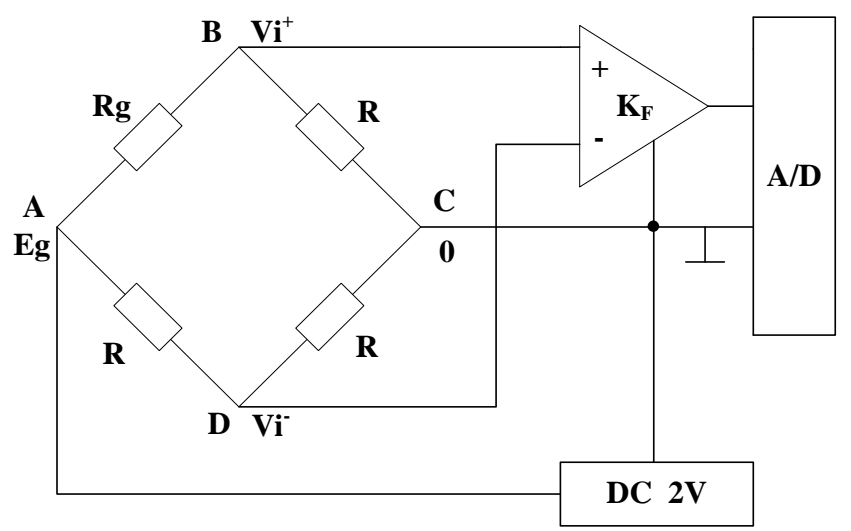

Figure 1 Stress test principle

Where, $R_{g}$ was the strain gauge resistor, $R$ was the fixed resistor.

According to the direct current bridge principle, it can be obtained.

$V_{i}=\frac{1}{4} E_{g} K \varepsilon, \quad V_{o}=K_{F} V_{i}=\frac{1}{4} K_{F} E_{g} K \varepsilon$

Then

$$
\varepsilon=\frac{4 V_{o}}{K_{F} E_{g} K}
$$

Where, $V_{i}$ was the output voltage of the direct current bridge. $E_{g}$ was the bridge voltage. $K$ was the sensitivity factor of the strain gauge. $\varepsilon$ was the input strain. $V_{O}$ was the output voltage of the low drift differential amplifier. $K_{F}$ was the gain of the low drift differential amplifier

\section{Data Acquisition System}

Data acquisition system DH5922 was used to measure the dynamic parameters. The system not only contained signal conditioners, direct current voltage amplifiers, A/D converters, anti-alias filters and so on, but also convenient control software and analysis software. The system consisted of 16 channels, and each channel had the maximum data acquisition frequency of $50 \mathrm{kHz}$.

\section{Test data processing method}

In the test, the signal was always affected by external factors. The signal often contained the high-frequency noise and low-frequency trend term. The high frequency noise was mainly caused by the impact among gun parts, AC noise of devices and so on. The low frequency trend term was caused by the zero drift of instruments, inappropriate capture cost, inaccuracy zero setting of instruments and other factors ${ }^{[4]}$.

According to characteristics of the signals, the combination method of low pass filter and empirical mode decomposition (EMD) was adopted to filter test data, and to eliminate or weaken the noise interference. In other words, low pass filter was used to filter high frequency noise and EMD was used to resolve and remove the effect of trend term. Detail steps were show in Figure 2.

$$
\begin{aligned}
& \text { Low pass Empirical mode } \\
& x(t) \underset{\text { (1) }}{\stackrel{\text { filering }}{\longrightarrow}} x_{1}(t) \stackrel{\text { decomposition }}{\longrightarrow} \sum_{i=1}^{n} C_{i}(t)+R(t) \underset{\text { (2) }}{\stackrel{\text { selection }}{\longrightarrow}} \sum_{j=1}^{m} C_{j}(t)
\end{aligned}
$$

Figure 2 Test data filtering process

\section{Low-pass filtering}

Low-pass filtering process of test data usually consisted of the DC removal, fast fourier 
transform, high frequency cutting off, inverse fourier transform and so on. Through the low-pass filtering, the high frequency noise was filtered, and the original test data series $x(t)$ was transformed to $x_{1}(t)$.

\section{Empirical mode decomposition}

EMD was a time-frequency analysis method that was put forward by Huang, which decomposed the data signal based on its time length ${ }^{[5]}$. In the theory, EMD had an obvious advantage in aspect of non-stationary and nonlinear data processing ${ }^{[6]}$. The original time series can be resolved to the intrinsic mode function (IMF) components and the residual signal. According to the characteristics of signal frequency and the trend term meaning, the IMF components corresponded to the trend term and the residual signal component were removed., the left $m$ IMF components was summed to obtain the valid signal.

\section{Test data curves}

The dynamic parameters were measured and filtered on live firing condition. Test curves of recoil displacement, recoil velocity, recoil resistance and gun carriage stress were obtained, shown in Figure 3 to Figure 6. Test results of gun dynamic parameters can provide a data validation for the gun virtual prototype model and a theoretical reference for the gun structure design.

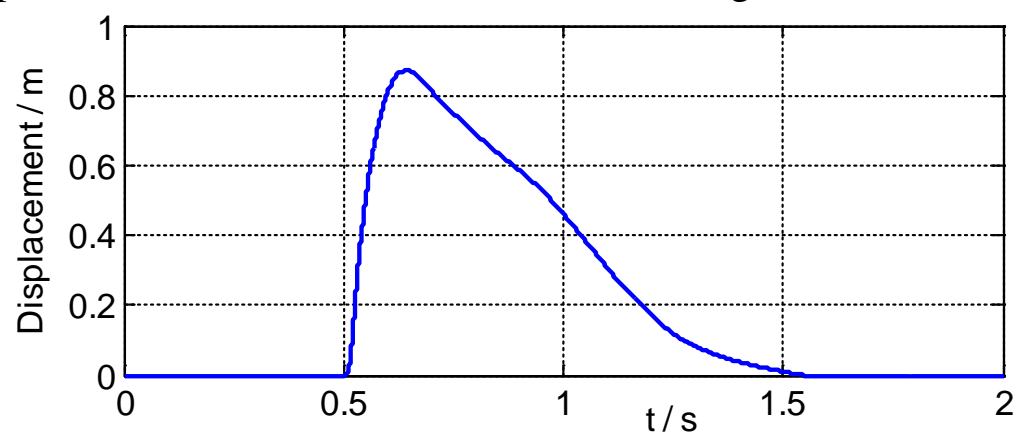

Figure 3 Test curve of recoil displacement

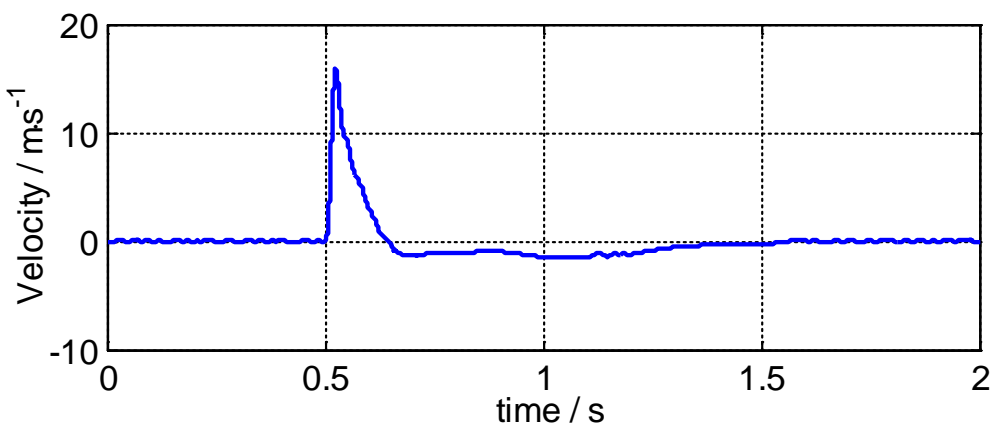

Figure 4 Test curve of recoil velocity

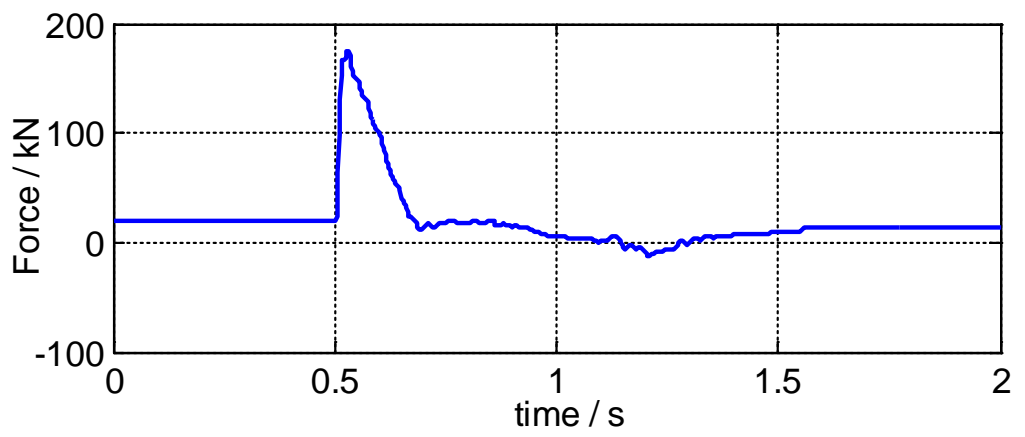

Figure 5 Test curve of recoil resistance 


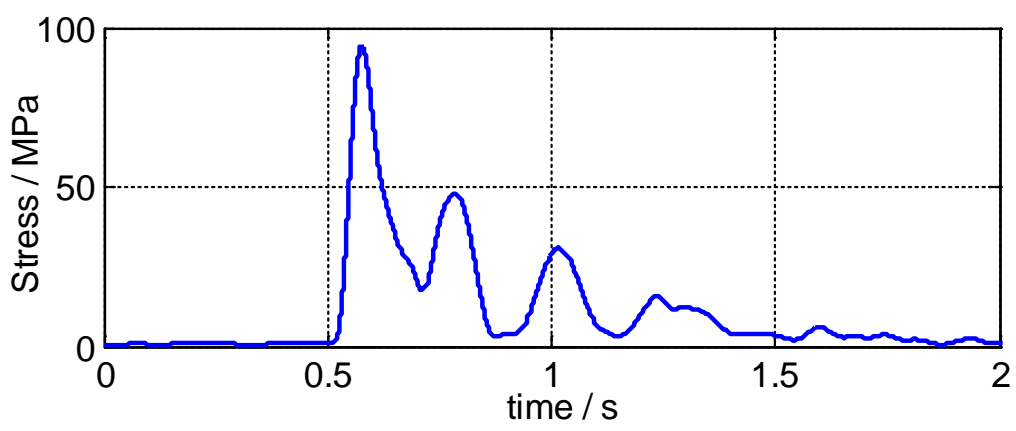

Figure 6 Test curve of gun carriage stress

\section{Conclusions}

The main research contents of this paper were

(1) According to the characteristics of dynamic parameters, measurement programs were developed respectively. Recoil displacement was measured by the cable-type displacement sensor. Recoil resistance was measured and calculated by the pressure gage, piezoelectric pressure sensor and quadrant. Gun carriage stress was measured by the resistance strain gauge.

(2) Test data of dynamic parameters was filtered by the low-pass filtering and EMD method. This article can provide a method reference for gun measurement technology of dynamic parameter.

\section{References}

[1] JIN Xiu-wen. Measurement technology of gun dynamic test [M]. Beijing: National defend industry press, 2004.

[2] LVU Shi-le, FU Jian-ping, ZHANG Li-hua, et al. Parameter test and disposal on gun firing with liquid projectile [J]. Journal of sichuan ordnance, 2014, 35(5): 47-50.

[3] ZHANG Xiang-yan, ZHENG Jian-guo, YANG Jun-rong. Gun design theory [M]. Beijing: Beijing institute of technology press, 2005.

[4] XU Yong, OU Yong-peng, DONG Wen-cai. Study on the measured signal analysis method based on low-pass filtering and EMD for ship seakeeping test [J]. Journal of ship mechanics, 2009, 13(5): 712-717.

[5] LI Hui-hao, XU Bao-jie, ZUO Yun-bo, et al. The comparative study of the signal trend extraction based on wavelet transformation and EMD method [J]. Instrumentation analysis monitoring, 2013, (3): 28-30.

[6] WANG Ruo-ping, YANG Yan-peng, WANG Guo-lin, et al. Tractor and farm transporter [J]. Application of EMD to road roughness trend, 2010, (4): 64-66. 